\title{
Advances in Multi-Signal Spectrum-Image Acquisition and Data Extraction Techniques involving Spectral Cathodoluminescence
}

\author{
S. A. Galloway and P. J. Thomas
}

Gatan UK, 25 Nuffield Way, Abingdon, Oxon, OX14 1RL, UK

The complementary nature of the various signals that may be captured in the SEM or STEM has stimulated advances in the acquisition and data extraction techniques for Spectrum-Imaging. In the case of Cathodoluminescence (CL), a multi-signal acquisition approach, for example to acquire an EDS spectrum simultaneously, is useful especially since the phenomena governing light generation can be complex to understand. Here we report on improvements to the methodology of multi-signal, spectrum-image acquisitions and post processing steps for extraction of meaningful data.

Multi-signal acquisition benefits from a single "master" control platform. This removes uncertainties associated with positional synchrony and helps avoid unwanted beam induced changes or quenching effects associated with sequential acquisitions. A simple and commercially viable method to realize a master platform approach is to employ one digital beam control and acquisition system, and acquire raw data from a $3^{\text {rd }}$ party operating system using common computer network protocols. When considering the experimental setup for multi signal acquisitions, the solid angle posed to the different detectors needs consideration. In addition there is the question of balancing injection levels, and detector binning periods specific to the different acquisition techniques.

With spectrum-imaging, the pixel dwell time can be long compared to standard imaging techniques, e.g. 20-2000ms. An active drift compensation routine can enhance the spatial resolution integrity of the data sets. However, the necessary dwell time tends preclude high resolution maps. Low density maps, especially at lower magnifications, are often associated with statistical under-sampling as the beam interaction volume is smaller than the grid point spacing. Furthermore with CL, the filling and emptying of opto-electronic traps is a dynamic process such that the light emission can be a function of the scanning speed. In this way the CL signal and possible signal quenching effects, can be strongly influenced by the pixel dwell time, with longer dwell times not necessarily providing the requisite improved signal to noise. A useful tool in both respects is sub-pixel scanning in which the focused probe is actively scanned across many defined points per grid position. This approach is also beneficial in order to minimize beam damage. An additional enhancement to the acquisition process is to acquire the SEM image in synchrony with the spectrum image. This provides confidence to the positional integrity of long acquisition time data sets.

Simultaneously acquired EDS and CL spectrum-images contain a wealth of information, and one data set can support the interpretation of the other. A simple but important example is a semiconductor alloy where full characterization of the specimen means differentiating spectral features associated with local variations in the alloy makeup to that of other effects governing the radiative recombination processes [1]. Peak fitting software allows the data set to be condensed into three, simple to understand maps; the peak wavelength, the peak width and the amplitude. As normal CL images tend to concentrate solely on the amplitude, the benefits of extracting peak position and width information is an important characterization capability. 
For more complex specimens, for example associated with geological applications, supporting mineralogical phase information is especially useful in aiding spectral CL interpretation and vice versa. For such applications, a Multi-Linear Least Squares (MLLS) algorithm helps extract phase information which is often not apparent using simple bandpass imaging or extraction tools. With this algorithm the user can specify any number of reference "finger-print" spectra, either taken from part of the spectrum image, or from another specimen or source. The algorithm then provides a fit-coefficient map of each of these reference spectra to the parent spectrum image. This algorithm applies to EDS and CL data sets and is a useful tool for working with complex spectral features since the information hidden one or more 3D data stacks can be condensed into a few simple phase maps [2].

\section{References}

[1] P. R. Edwards, R. W. Martin, K. P. O’Donnell and I. M. Watson, phys. stat. sol. (c) 0, No. 7 (2003) 2474

[2] Jacob Wilbrink, Gatan Inc. is gratefully acknowledged for his contribution to this work.
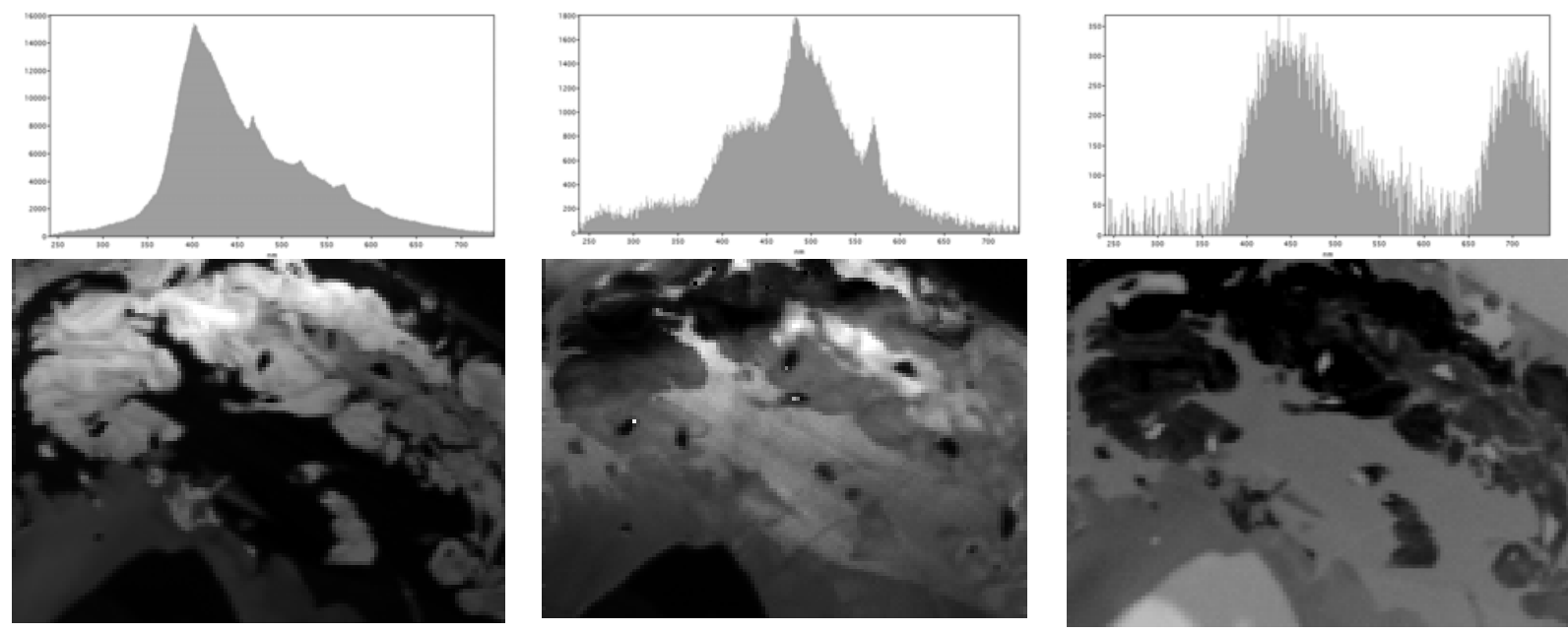

20microns

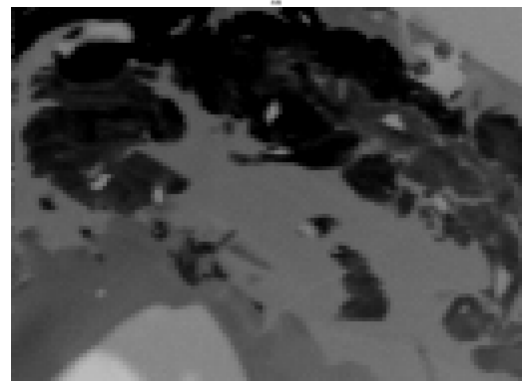

Fig. 1. Selected spectra and MLLS extractions from a CL spectrum-image of a polished zircon specimen. The bottom intensity normalized images are fit-co-efficient maps of each of the three chosen spectra shown above. 